\title{
Erratum
}

\section{A Model for Generalization and Specification by Single Neurons}

Paul W. Munro

Center for Neural Science, Brown University, Providence, RI 02912, USA

Biol. Cybern. 51, 169-179 (1984)

In Sect. 2.2.2, the paragraph following the theorem (end of p. 171) should be corrected by interchanging the words "positive" and "negative." The corrected version reads as follows (corrections underscored):

The (constant) sign of $W(x)$ will be shown to determine the feature abstracting property of the neuron. If $W(x)$ is always negative, the neuron generalizes (a "G-cell") and if it is positive the neuron tunes to a specific stimulus (an "S-cell"). 